\title{
ASSOCIAÇÃO ENTRE DESEMPENHO DAS FUNÇÕES CORTICAIS E ALFABETIZAÇÃO EM UMA AMOSTRA DE ESCOLARES DE PRIMEIRA SÉRIE DE PORTO ALEGRE
}

\author{
ANA GUARDIOLA*, LUCIA TERESINHA CUNHA FERREIRA **, NEWRA TELLECHEA ROTTA***
}

\begin{abstract}
RESUMO - As funções corticais constituem a base do processo de aprendizado e o exame neurológico evolutivo (ENE) avalia o nível de desenvolvimento em que elas se encontram. O objetivo desta pesquisa foi estudar, através do ENE, as funções corticais de uma população representativa da 1. ${ }^{a}$ série de Porto Alegre e investigar seu desempenho no aprendizado. Efetuou-se estudo observacional, analítico e de delineamento transversal. A amostra foi aleatória, constituída de 484 crianças. Avaliaram-se sexo, idade, cor, peso, estatura, exame neurológico, exame neurológico evolutivo, desempenho nos subtestes números, completar figuras e código da escala de WISC e alfabetização. Os itens do ENE encontravam-se abaixo do esperado para idade em 11,4\% e 38,2\% das crianças; o mais atingido foi sensibilidade e gnosias $(38,2 \%)$ e o menos comprometido foi persistência motora $(11,4 \%)$. Nos testes psicométricos, $40,5 \%$ tinham desenvolvimento abaixo do esperado em números, 39,0\% em completar figuras, $71,5 \%$ em código. Quanto a alfabetização, $94,4 \%$ das crianças se alfabetizaram. A disfunção neuropsicológica e a lateralidade mal estabelecida foram fatores de risco para o aprendizado.
\end{abstract}

PALAVRAS-CHAVE: funções corticais, exame neurológico evolutivo, alfabetização.

\section{Performance of literacy and cortical brain functions in a sample of first grade students of Porto Alegre, Brazil}

ABSTRACT - Cortical brain functions are the basis of the learning process and evolutionary neurological examination (ENE) provides the level of development of the brain. The purpose of this work was to study by means of the ENE the brain funtions in a representative sample of first grade students of Porto Alegre, to investigate the learning performance. An observation, analytical and transversal delineated study was performed. The random sample had 484 children. It was observed: sex, age, race, weight, height, neurological examination, ENE, performance in the number sub-tests, completing figures and codes from WISC scale and literacy learning. The items of ENE were below the expected for the age in $11.4 \%$ and $38.2 \%$ of the children: the most affected ones were the sensitivity activity and gnosias $(38.2 \%)$ and the least one was motor persistence (11.4\%). In psychometric tests, $40.5 \%$ of the subjects had their development in numbers below the espected, $39.0 \%$ in accomplishing figures, $71.5 \%$ in codes. Concerning literacy, $94.4 \%$ of the children learned to read and write. The neuropsychological dysfuntion and malestablished laterality were a risk factor for the learning process.

KEY WORDS: brain functions, evolutionary neurological examination, literacy.

O estudo dos processos corticais traz informações de valor inestimável e revela importantes caminhos para o conhecimento do sistema funcional complexo que é o cérebro. O sistema nervoso, concebido como um ordenador, assegura a integração das informações que recebe do mundo exterior e do próprio organismo, endereçando, finalmente, de forma coordenada, aos órgãos efetores, as ordens necessárias à vida do indivíduo (motricidade voluntária, funções psíquicas, respiração, digestão,

*Livre Docente em Neurologia Infantil e Professora Adjunta da Fundação Faculdade Federal de Ciências Médicas de Porto Alegre (FFFCMPA); **Doutoranda da FFFCMPA; *** Livre Docente em Neurologia e Professora Adjunta da Universidade Federal do Rio Grande do Sul. Aceite: 7-janeiro-1998.

Dra. Ana Guardiola - Departamento de Neurologia, FFFCMPA - Rua Sarmento Leite 245 - 900501-170 Porto Alegre RS - Brasil. 
circulação sanguínea e sobrevivência da espécie). O cérebro, com seus dois hemisférios, funciona como um todo, estruturando a conduta do indivíduo em seus aspectos cognitivo e afetivo. Ambos os hemisférios estão integrados pela presença das comissuras inter-hemisféricas, e o córtex cerebral está relacionado, através das vias de associação, às estruturas subcorticais: corpo estriado, tálamo óptico, tronco encefálico, medula e cerebelo. Neste contexto, Luria ${ }^{1}$ descreve três unidades funcionais:

1. Primeira unidade funcional ou de vigília -. As estruturas do tronco cerebral que participam do controle do ciclo sono-vigília são o sistema reticular ascendente, representadas fundamentalmente pelos núcleos colinérgicos, noradrenérgicos, dopaminérgicos e serotoninérgicos. O córtex cerebral também participa, especialmente o córtex pré-frontal. A alteração anatômica ou funcional deste sistema produz diversas alterações clínicas, desde distração até síndrome comatosa.

2. Segunda unidade funcional ou de recepção. É a área da análise e do armazenamento da informação, representada pelos córtices temporal, parietal e occipital, existindo as áreas primárias, secundárias e terciárias.

As áreas primárias são aquelas onde terminam as fibras sensitivas que provêm do tálamo. No lobo temporal estão as áreas auditivas primárias 41 e 42, no primeiro giro temporal superior; no lobo parietal está a área somestésica que ocupa o giro pós-central; e no lobo occipital, na face interna, a área visual 17. As áreas primárias só registram os elementos da experiência, sem ter caráter simbólico.

As áreas secundárias se situam junto às primárias. A área 22 é a área auditiva secundária; as áreas 18 e 19 constituem a área visual; e as áreas 5 e 7 do lobo parietal, a área somestésica. A principal função destas áreas é processar a informação que chega às áreas primárias e dar-lhes conteúdo simbólico.

As áreas terciárias não têm localização tão precisa. São áreas de associação entre as áreas secundárias, integrando experiência multissensorial. Dessa forma se integram funções complexas, como a linguagem, esquema corporal, espaço, espaço-tempo e cálculo, entre outras. $\mathrm{O}$ desenvolvimento destas áreas determina o estabelecimento do hemisfério esquerdo em relação à linguagem.

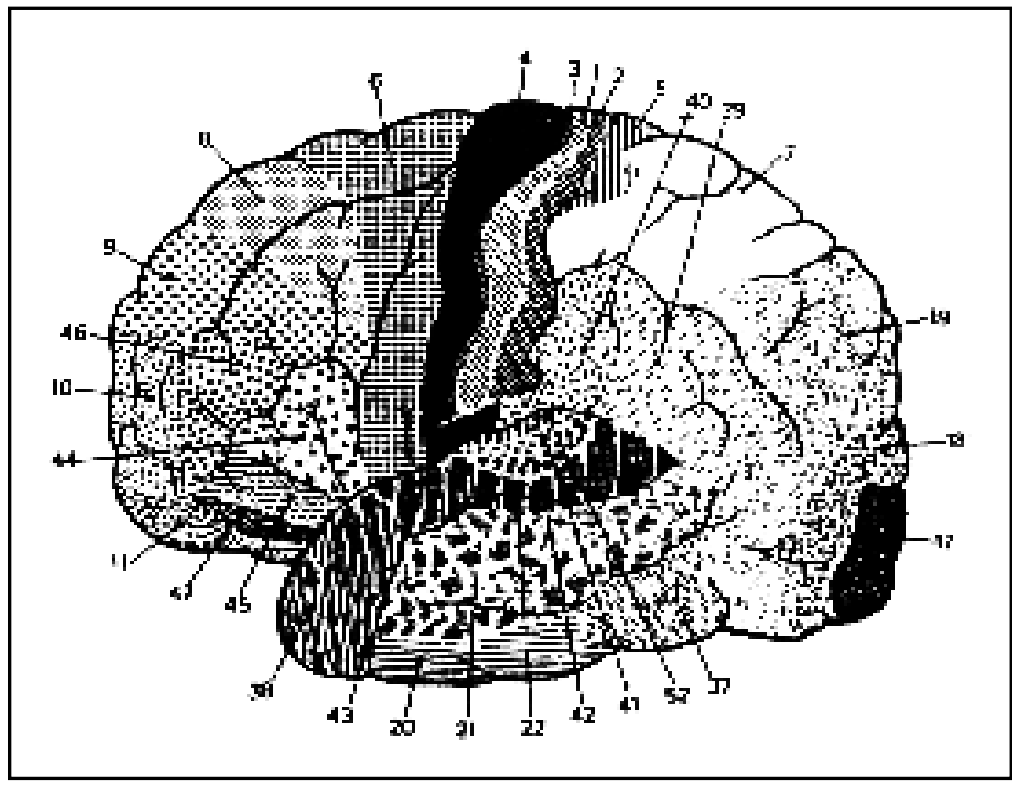

Fig 1. Áreas hemisféricas segundo Brodmann. 


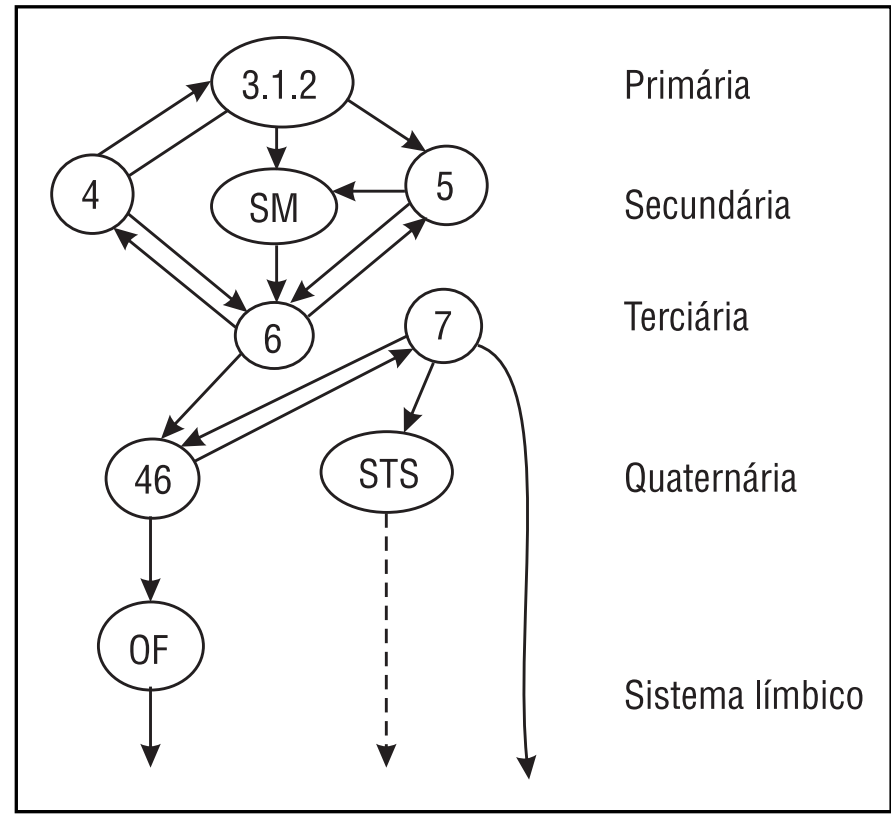

Fig 2. Áreas somestésicas primárias, secundárias, terciárias e quaternárias (Jones \& Powel, 1970)․․

3. Terceira unidade de programação, regulação e verificação da atividade. É representada pelos lobos frontais, que tornam possível a intencionalidade, a planificação e a organização da conduta em relação a percepção e ao conhecimento do mundo (Fig 1).

Jones \& Powell ${ }^{2}$ aceitam a existência de áreas primárias, secundárias, teciárias, quaternárias e quinquenárias (Fig 2).

Rebollo $^{3}$ incorpora uma quarta unidade funcional, representada pelo sistema límbico, que intervém na seleção dos estímulos segundo suas características e tonalidade afetiva, e a porção orbitária do lobo frontal que participa na planificação da conduta no seu aspecto afetivo. A dominância hemisférica é controlada pelos centros da linguagem que, na maioria das pessoas, se localiza no hemisfério esquerdo, ou dominante, ou maior. Atualmente se atribui ao hemisfério direito um papel importante na percepção do esquema corporal e do espaço gráfico e da construção, já que o hemisfério direito predomina na função de perceber e analisar os modelos visuais. O hemisfério esquerdo predomina em relação à linguagem, mas o direito é mais importante para a percepção das melodias. Também se notam as diferenças entre ambos os hemisférios em relação à memória; o esquerdo está relacionado a memória verbal, e o direito com as localizações espaciais, fisionomias e melodias ${ }^{4}$. As praxias, gnosias e linguagem, funções corticais altamente elaboradas, se inserem nesse contexto ${ }^{5}$.

O aprendizado é processo complexo, dinâmico, estruturado a partir de um ato motor e perceptivo, que, elaborado corticalmente, dá origem à cognição. Os distúrbios de áreas específicas do sistema nervoso central (SNC), relacionadas com a noção do esquema corporal, do espaço e do tempo, constituem as bases neuropatológicas das alterações perceptomotoras ou dispatognósicas, das quais poderiam resultar os quadros de dislexia, disgrafia e discalculia ${ }^{6}$.

As altas taxas de reprovações em escolares que ingressam no primeiro ciclo têm despertado a atenção dos especialistas que atendem crianças em idade escolar, incluindo pedagogos, psicólogos, pediatras, neurologistas e psiquiatras ${ }^{7-9}$. Sabe-se que o aprendizado pode ser afetado por: fatores 
genéticos, afecções sensoriais periféricas (surdez, cegueira), afecções neurogênicas, retardo mental, afecções médicas gerais, como desnutrição e doenças crônicas; desvantagem sócio-cultural (falta de oportunidade, escassa motivação para aprender, lar desorganizado); e transtornos pedagógicos (número excessivo de alunos, inassistência à classe, didática inadequada) ${ }^{10-14}$.

Nesta linha, os transtornos de aprendizado têm múltiplos enfoques: neurológico, psicológicos, pedagógicos e sociais. Dentro do enfoque neurológico, tem que se salientar que, para que exista o ato de aprender, é necessário que o indivíduo tenha condições para tal. $\mathrm{O}$ ato complexo de aprender pode ser resumido, rapidamente, a partir das aferências sensitivas que chegam ao córtex cerebral, que se mantêm no estado de alerta a partir da ação que exerce sobre ele a substância reticular ativadora ascendente. $\mathrm{O}$ estado de alerta é fundamental na aprendizagem, permite ao córtex selecionar os diversos estímulos que recebe e programar a resposta desejada. $\mathrm{O}$ afeto, representado aqui pela função do hipotálamo, interfere na reação em cadeia que se estabelece a partir do estímulo sensitivosensorial e termina com a atividade motora (Fig 3$)^{13}$.

Dentro das perturbações neurogênicas que podem produzir alterações no aprendizado, temos os transtornos das integrações funcionais básicas (visomotora e auditivo-visual), do esquema corporal, da orientação espacial, do ritmo, da coordenação motora fina, da lateralidade, da função análise e síntese, da integração das partes em um todo, da simbolização, da linguagem em seus diferentes aspectos, dos impulsos, da atenção e da memória ${ }^{5,10,13,15-17}$.

As funções corticais constituem, portanto, a base de qualquer processo de aprendizado, e o exame neurológico evolutivo (ENE), de acordo com os critérios e a técnica estabelecidos por Lefèvre ${ }^{18}$, proporciona o nível de desenvolvimento em que elas se encontram.

O objetivo deste estudo foi estudar através do ENE e dos subtestes números, completar figuras e código do WISC, as funções corticais de uma população representativa de 1. ${ }^{a}$ série de Porto Alegre, correlacionando variáveis clínicas com sua alfabetização.

\section{MÉTODOS}

Foi realizado estudo observacional, analítico e de delineamento transversal, sendo utilizada amostragem aleatória e proporcional de todos os escolares $(n=35.521)$ da $1^{\mathrm{a}}$ série, distribuídos em escolas estaduais $(n=22.966)$, particulares $(n=8.311)$ e municipais $(n=4.244)$.

O tamanho da amostra foi calculado estimando uma precisão de medida $\pm 3,0 \%$, correspondendo ao intervalo de confiança de $95 \%$, para uma prevalência prevista de $10 \%$. Assim, a amostra ficou

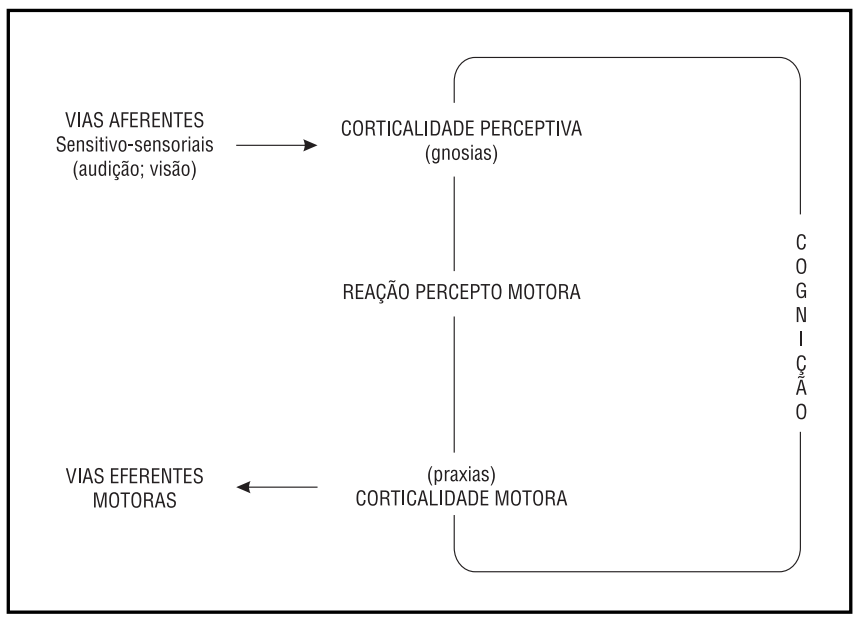

Fig 3. Aspectos neurológicos da aprendizagem (Rotta, 1988) $)^{13}$. 
constituida de 310 crianças da rede estadual, 58 da rede municipal e 116 crianças das escolas particulares, totalizando 484 crianças. Aferiram-se sexo, idade, cor, estado nutricional, escola de origem.

$\mathrm{O}$ exame neurológico foi feito em todas as crianças, avaliando-se: psiquismo, linguagem, fácies, atitude, equilíbrio, tono, motricidade voluntária, motricidade reflexa, motricidade passiva, sensibilidade e nervos cranianos. Definiu-se como normal o exame que tinha, no máximo, alterações do tono e da linguagem. As crianças que não preencheram este critério foram excluídas.

O ENE, ao avaliar linguagem, lateralidade, equilíbrio estático, equilíbrio dinâmico, coordenação apendicular, persistência motora, sensibilidade e gnosias, coordenação tronco-membros, foi usado para aferir maturidade neurológica. Quando a criança não conseguiu realizar as provas do ENE para a sua faixa etária, de acordo com os critérios e a técnica estabelecidos por Lefèvre, foi considerada abaixo do normal.

Aplicaram-se também subtestes números, completar figuras e código da escala de WISC.

A alfabetização (sim ou não) foi informada pela professora.

Foi obtido consentimento das Secretarias de Educação do Estado e do Município e da Direção das escolas particulares.

As crianças foram examinadas individualmente na própria escola. O tempo usado para avaliação foi em torno de 60 minutos.

Aplicou-se o teste qui-quadrado para verificar a significância das associações entre variáveis categóricas. Usou-se a ANOVA para variáveis contínuas. Considerou-se o P alfa 5\% significante (bicaudal).

Testou-se associação bruta de sexo, idade, cor, tipo de escola, com ENE e alfabetização.

\section{RESULTADOS}

Todas as crianças apresentaram exame neurológico normal exceto no tono muscular, em que havia $4(0,8 \%)$ crianças com hipotonia e $35(7,2 \%)$ com paratonias.

A Tabela 1 mostra a distribuição das crianças por escola e por sexo e a Tabela 2 a idade, peso e estatura. Na Tabela 3 observa-se distribuição por cor.

A Tabela 4 mostra a associação entre funções do ENE e alfabetização

Nos subtestes números 196 crianças (40,5\%), completar figuras 189 crianças (39\%) e códigos 346 crianças $(71,5 \%)$ apresentaram índices abaixo da média ponderada. Quando se considerou o desempenho psicométrico alterado globalmente, haviam 82 crianças $(16,9 \%)$ mostrando associação com os distúrbios da alfabetização (Tabela 5).

A Tabela 5 mostra que houve associação entre a lateralidade mal estabelecida e dificuldade de alfabetização.

Tabela 1. Distribuição das crianças por escola e por sexo.

\begin{tabular}{|c|c|c|c|c|c|c|}
\hline \multirow[t]{2}{*}{ Escola } & \multicolumn{2}{|c|}{ Meninos } & \multicolumn{2}{|c|}{ Meninas } & \multicolumn{2}{|c|}{ Total } \\
\hline & $\mathrm{N}$ & $\%$ & $\mathrm{~N}$ & $\%$ & $\mathrm{~N}$ & $\%$ \\
\hline Estadual & 145 & 46,8 & 165 & 53,2 & 310 & 64,7 \\
\hline Municipal & 25 & 43,1 & 33 & 56,9 & 58 & 11,9 \\
\hline Particular & 69 & 59,5 & 47 & 40,5 & 116 & 23,4 \\
\hline Total & 239 & 49,4 & 245 & 50,6 & 484 & 100 \\
\hline
\end{tabular}

$\mathrm{X}^{2}=6,49 \mathrm{P}=0,039^{*}$

* P com significação estatística. 
Tabela 2. Idade, peso, estatura por sexo (média, DP).

\begin{tabular}{lccccc}
\hline Características & Amostra & Meninos & Meninas & $\mathrm{F}$ & $\mathrm{P}$ \\
\hline Idade (meses) & 89,5 & 90,3 & 88,3 & 4,37 & $0,038^{*}$ \\
& $(11,0)$ & $(11,9)$ & $(9,9)$ & & \\
Peso (kg) & 26,1 & 26,1 & 26,0 & 0,04 & 0,839 \\
& $(5,6)$ & 5,8 & $(5,5)$ & & \\
Estatura (cm) & 126,8 & 127,0 & 126,6 & 0,23 & 0,632 \\
& $(7,3)$ & $(7,8)$ & $(7,9)$ & & \\
\hline
\end{tabular}

* P com significação estatística.

Tabela 3. Cor das crianças matriculadas nas escolas.

\begin{tabular}{|c|c|c|c|c|c|}
\hline \multirow[t]{2}{*}{ Cor } & \multicolumn{3}{|c|}{ Escola } & \multirow[t]{2}{*}{$X^{2}$} & \multirow[t]{2}{*}{$\mathrm{P}$} \\
\hline & Estadual & Municipal & Particular & & \\
\hline Branca & $\begin{array}{c}258 \\
(83,2)\end{array}$ & $\begin{array}{c}37 \\
(63,8)\end{array}$ & $\begin{array}{c}105 \\
(90,5)\end{array}$ & 19,46 & $<0,001 *$ \\
\hline Não branca & $\begin{array}{c}52 \\
(16,8)\end{array}$ & $\begin{array}{c}21 \\
(36,2)\end{array}$ & $\begin{array}{c}11 \\
(9,5)\end{array}$ & & \\
\hline Total & $\begin{array}{c}310 \\
(100 \%)\end{array}$ & $\begin{array}{c}58 \\
(100 \%)\end{array}$ & $\begin{array}{c}116 \\
(100 \%)\end{array}$ & & \\
\hline
\end{tabular}

* P com significação estatística.

Tabela 4. Associação entre funções do ENE e alfabetização das crianças.

\begin{tabular}{|c|c|c|c|c|c|}
\hline \multirow[t]{2}{*}{ Funções } & & \multicolumn{2}{|c|}{ Não alfabetizadas } & \multirow[t]{2}{*}{$\mathrm{X}^{2}$} & \multirow[t]{2}{*}{$\mathrm{P}$} \\
\hline & & $\mathrm{N}$ & $\%$ & & \\
\hline Equilíbrio estático & $\begin{array}{c}\text { A (80) } \\
\text { N (404) }\end{array}$ & $\begin{array}{l}13 \\
14\end{array}$ & $\begin{array}{l}16,3 \\
3,5\end{array}$ & 18,36 & $<0,001 *$ \\
\hline Equilíbrio dinâmico & $\begin{array}{c}\text { A (61) } \\
\text { N (423) }\end{array}$ & $\begin{array}{l}12 \\
15\end{array}$ & $\begin{array}{c}19,7 \\
3,5\end{array}$ & 23,34 & $<0,001 *$ \\
\hline Coordenação apendicular & $\begin{array}{l}\text { A (146) } \\
\text { N (338) }\end{array}$ & $\begin{array}{c}23 \\
4\end{array}$ & $\begin{array}{c}15,8 \\
1,2\end{array}$ & 38,37 & $<0,001 *$ \\
\hline Persistência motora & $\begin{array}{c}\text { A (55) } \\
\text { N (429) }\end{array}$ & $\begin{array}{c}9 \\
18\end{array}$ & $\begin{array}{c}16,4 \\
4,2\end{array}$ & 11,49 & $<0,001 *$ \\
\hline Sensibilidade e gnosias & $\begin{array}{l}\text { A (185) } \\
\text { N (299) }\end{array}$ & $\begin{array}{l}17 \\
10\end{array}$ & $\begin{array}{l}9,2 \\
3,3\end{array}$ & 6,34 & 0,011 \\
\hline Coordenação tronco-membros & $\begin{array}{c}\text { A (79) } \\
\text { N (405) }\end{array}$ & $\begin{array}{l}15 \\
12\end{array}$ & $\begin{array}{c}19 \\
3\end{array}$ & 29,26 & $<0,001 *$ \\
\hline Linguagem & $\begin{array}{c}\mathrm{D}(30) \\
\mathrm{N}(454)\end{array}$ & $\begin{array}{c}7 \\
20\end{array}$ & $\begin{array}{c}23,3 \\
4,4\end{array}$ & 15,71 & $<0,001 *$ \\
\hline
\end{tabular}

A, alterado; $\mathrm{N}$, normal; D, dislalias.

* P com significação estatística. 
Tabela 5. Associação entre lateralidade e processo de alfabetização.

\begin{tabular}{lrcccc}
\hline Lateralidade & & Não alfabetizadas & $\%$ & $X^{2}$ & $P$ \\
\hline Direita & $(248)$ & 16 & 6,5 & 13,42 & $0,003^{*}$ \\
Esquerda & $(12)$ & 0 & 0 & & \\
Cruzada & $(199)$ & 6 & 3,0 & & \\
Mal estabelecida & $(25)$ & 5 & 20,0 & & \\
\hline
\end{tabular}

* P com significação estatística.

Estes resultados mostram que houve associação entre as funções corticais alteradas, a lateralidade mal estabelecida e os distúrbios na alfabetização $(p<0,01)$.

\section{DISCUSSÃO}

A literatura assinala que crianças com distúrbios da aprendizagem, da atenção e neuropsicológicos não apresentam alterações significativas no exame neurológico clássico ${ }^{9,11,19-21}$. Neste estudo os dados concordam com a literatura referida já que não se encontraram alterações exceto no tono muscular. Havia hipotonia em $0,8 \%$ das crianças e paratonias em $7,2 \%$ o que, como único sinal, não constitui manifestação patológica.

Em relação a lateralidade, assunto controvertido, os achados desta investigação concordam com a opinião de que a lateralidade mal estabelecida pode resultar em problemas de linguagem ou dificuldades escolares ${ }^{9,11,22,23}$.

O conhecimento do perfil neuropsicológico de uma criança é de extremo valor quando se estudam distúrbios do aprendizado ou do comportamento.

A linguagem é a função mais elaborada e complexa do SNC. Pesquisadores relatam maior incidência de dificuldades na escrita e na leitura em crianças que apresentam atraso na aquisição da linguagem falada ${ }^{20,21,25}$. Lefèvre observou, na sua pesquisa sobre o ENE, que as crianças normais, aos 3 anos, haviam superado a etapa de palavra frase e as dislalias por troca, podendo apresentar somente dislalias por supressão ${ }^{18}$. Rotta salienta que uma criança em idade escolar não deve apresentar alterações verbais e que a presença destas alterações implica maior probabilidade de a criança apresentar dificuldades escolares ${ }^{9}$. Neste estudo só 30 crianças apresentaram dislalias por supressão, alterações da linguagem verbal que indicaram padrão evolutivo abaixo do normal, e 7 (23,3\%) mostraram maior dificuldade na alfabetização.

O equilíbrio estático é função neurológica importante para a manutenção de posturas adequadas, imprescindíveis no ato de aprender e no comportamento ${ }^{5,9,19,21}$.

O equilíbrio dinâmico, sendo também uma função evolutiva, proporciona indicativos de maturidade neurológica. Rotta ${ }^{9}$ Cypel $^{8}$ relatam que crianças com imaturidade desta função têm mais probabilidade de apresentar dificuldades de aprendizado.

Através das provas de coordenação apendicular e sensibilidade e gnosias avaliam-se importantes funções práxicas e gnósicas, sendo estas fundamentais no aprendizado. Na presença de dificuldades do aprendizado existe maior probabilidade destas áreas estarem alteradas ${ }^{5,6,9,16}$.

A persistência motora indica o padrão neurológico capaz de manter uma postura adequada, controlar os impulsos e possibilitar uma atenção sustentada. Quando alterada, associa-se a problemas de comportamento e de escolaridade ${ }^{11,13}$.

Neste estudo as crianças que apresentaram dificuldades de alfabetização mostraram associação com todas as funções do ENE alteradas.

O subteste números da escala de WISC estuda a atenção e a memória imediata. O subteste completar figuras dessa escala avalia, além da atenção e concentração, a habilidade para diferenciar detalhes essenciais dos não essenciais. O subteste código, por depender da atividade visual, da atividade motora, da coordenação e aprendizado, é considerado como um teste de velocidade 
psicomotora ${ }^{26}$. Esta foi a razão da escolha destes três subtestes para complementar o ENE no estudo desta série, observando-se associação, quando alterados, com as dificuldades de alfabetização.

\section{CONCLUSÕES}

Foi possível descrever de forma apurada o estudo das funções corticais através do ENE em uma amostra representativa de escolares da $1 .^{\mathrm{a}}$ série.

1. Todas as crianças apresentaram exame neurológico normal exceto no item tono muscular, em que havia $4(0,8 \%)$ crianças com hipotonia e $35(7,2 \%)$ com paratonias, e na linguagem em que $30(6,2 \%)$ apresentavam dislalias.

2. No exame neurológico evolutivo, o item mais atingido foi sensibilidade e gnosias $(38,2 \%)$ e o menor comprometimento ocorreu para persistência motora $(11,4 \%)$, não se observando diferenças entre meninos e meninas.

3. Em relação aos testes psicométricos, $40,5 \%$ das crianças tinham desenvolvimento abaixo do esperado em números, 39,0\% em completar figuras, 71,5\% em código. Quando considerados globalmente, $82(16,9 \%)$ tinham desempenho abaixo do esperado.

4. Quanto a alfabetização, praticamente todas $(94,4 \%)$ se alfabetizaram.

5. Houve associação entre as funções alteradas do ENE e dos testes psicométricos no processo da alfabetização.

6. A lateralidade mal estabelecida foi fator de risco para alfabetização.

\section{BIBLIOGRAFIA}

1. Luria AR. The working brain. New York: Basic Books, 1973.

2. Jones EG, Powell TPS. An anatomical study of converging sensory pathways within the cerebral cortex of the monkey. Brain 1970;93:793-820.

3. Rebollo MA. Disfunções hemisféricas. An Neuropediatr Latinoamer 1991;1:1-19.

4. Barbizet J, Duizabo P. Manual de Neuropsicologia. São Paulo: Masson, 1985.

5. Rebollo MA, Cardús S. Semiologia del sistema nervioso en el niño: exploración del desarrollo neuropsíquico. Montevideo: Delta 1973.

6. Rotta NT, Guardiola A. Distúrbios de aprendizagem. In Diament A, Cypel S. Neurologia Infantil. 3.Ed. São Paulo: Ateneu, 1996:1062-1074.

7. Brunquell PJ, Russman BS, Lerer TJ. Pediatric neurologists opinions regarding the assessment of children with learning disabilities. Ann Neurol 1991;30:477.

8. Cypel S. Avaliação neurológica e de alguns aspectos do comportamento em crianças de 6 a 7 anos com e sem dificuldades no aprendizado. Tese de Livre Docência, Faculdade de Medicina da Universidade de São Paulo, São Paulo, 1983.

9. Rotta NT. Avaliação neurológica evolutiva, eletroencefalográfica e psicológica em crianças com rendimento escolar deficiente. Tese de Livre Docência, Fundação Faculdade Católica de Medicina, Porto Alegre, 1975.

10. Guardiola A. Psicomotricidade e distúrbios de atenção. An Soc Bras Terap Psicomot (Porto Alegre) 1988:53-57.

11. Hara H, Fukuyama Y. Partial imitation and partial sensorial agnosia in mentally normal children with convulsive disorders. Acta Pediatr Japon 1992;34:416-425.

12. Knights RM, Ivan LP, Ventureyra EC, et al. The effects of head injury in children on neuropsychological and behavioural functioning. Brain Inj 1991;5:339-351.

13. Rotta NT. Aspectos neurológicos de los problemas de aprendizaje. An Neuropediatr Latinoamer 1988;1:11-16

14. Schachter DC, Pless IB, Bruck M. Self-report of family histories of learning difficulties. Can J Psychiatry 1992;37:29-32.

15. Berninger VW, Rutberg J. Relationship of finger function to beginning writing: application to diagnosis of writing disabilities. Dev Med Child Neurol 1992;34:198-215.

16. Levy HB, Harper CR, Weinberg WA. A practical approach to children failing in school. Pediatr Clin N Am 1992;39:895-928.

17. Sandler AD, Watson TE, Footo M, Levine MD, Coleman WL, Hooper SR. Neurodevelopmental study of writing disorders in middle childhood. J Dev Behav Pediatr 1992;13:17-23.

18. Lefèvre AB. Exame neurológico evolutivo do pré-escolar normal. São Paulo: Sarvier, 1972.

19. Guardiola A. Distúrbio de hiperatividade com déficit de atenção: um estudo de prevalência e fatores associados em escolares de 1. ${ }^{\text {a }}$ série de Porto Alegre. Tese de Doutorado, Faculdade de Medicina da Universidade Federal do Rio Grande do Sul. Porto Alegre, 1994

20. Illingworth R. Delayed motor development. Pediatr Clin N Am 1968;15:569-572.

21. Lefèvre AB. Disfunção cerebral mínima: estudo multidisciplinar. São Paulo: Sarvier 1975.

22. Harris AJ. Lateral dominancy, directional confusion and reading disability. J Psychol 1957;44:283-294.

23. Ross G, Lipper E, Auld PA. Hand preference, prematurity and developmental outcome at school age. Neuropsychologia 1992;30:483-494.

24. Gessel A, Amatruda C. Diagnóstico del desarollo normal y anormal del niño. Buenos Aires: Paidós, 1946.

25. Ingram TTS. Specific developmental disorders of speech in childhood. Brain 1959;82:450-467.

26. Wechsler D. Escala de inteligência Wechsler para crianças WISC. Rio de Janeiro: Cepa, 1964. 